\title{
Behavioural management of paediatric patients
}

\author{
An investigation of paediatric dentists' attitudes towards parental accompaniment and behavioural management \\ techniques in the UK M. L. Crossley and G. Joshi Br Dent J 2002; 192: 517-521
}

\section{Objective}

Good behavioural management techniques are essential for treating children. Recent research has investigated parental and paediatric attitudes towards various behavioural management techniques. However, in the UK, very little is known about paediatric attitudes towards such issues. Accordingly, the aim of this investigation was to conduct a survey in order to provide insight into the current thinking of paediatric dentists across the UK.

Study design

A questionnaire was developed based on previous surveys investigating the attitudes of paediatric dentists towards various behavioural management techniques in Australia and the USA. Areas covered in the questionnaire included: attitudes towards parental accompaniment; attitudes regarding parental expectations and attitudes towards the use of various different child management techniques. The questionnaire was sent to all registered members of the British Society of Paediatric Dentistry $(n=304)$.

Results

245 responses (80.5\%) were returned, of which 218 provided valid and useable information (72\%). A majority of $80 \%$ of respondents supported parental accompaniment of the child during the course of treatment. ANOVA procedures revealed significant differences between acceptability of parental accompaniment and the year in which dentists had qualified $(\mathrm{p}<0.05)$; more recently qualified dentists were less accepting of parental accompaniment. Only a minority of dentists reported feeling pressured by parental expectations with regard to their child's treatment. However, an independent sample $t$-test revealed that male dentists experienced greater perceived conflict between dental and parental expectations than female dentists $(p<0.05)$. In terms of specific behavioural management techniques, the most favoured was 'tell-show-do' with 87\% of respondents citing this as their most commonly used method. Least acceptable were 'hand over mouth' techniques, followed closely by active restraint and papoose board. Conclusion

In general, paediatric dentists in the UK favoured less 'restraining' methods of behavioural management. There was widespread support for parental accompaniment in the dentaI operatory and a desire to work in participation with parents in order to facilitate the child's good behaviour and more effective dental treatment. Further studies comparing the effectiveness of various techniques used with regard to treatment time and clinical outcomes, are now required.

\section{IN BRIEF}

- Good behavioural management techniques are essential for providing effective child dental treatment. Currently, little is known in Britain about paediatric dentists' attitudes towards different behavioural techniques. This study provides preliminary insight into these attitudes.

- The study shows that the paediatric dentists surveyed were positively oriented to parental accompaniment of the child during treatment and generally viewed parents as useful allies in facilitating dental treatment for the child more effectively.

- Further studies, comparing the effectiveness of various behavioural management techniques, are now required.

\section{COMMENT}

Managing the anxious dental patient is difficult enough, but how about trying to treat a howling 3-year-old, who is clinging to his mother? For those of us who treat children, the 'dark art' of behaviour management is not always easy to explain. Even at the undergraduate level, there are those who are just inherently more successful in coaxing frightened or truculent children into the dental chair. It is also fascinating to observe how practitioners from other countries differ in their favoured management techniques: one of the most frequently cited examples being the penchant of North Americans for the 'papoose board!'

In order to elucidate British dentists' attitudes towards a variety of behaviour management techniques used for children, Crossley and Joshi chose to survey members of the British Society of Paediatric Dentistry. In particular, respondents were asked how they felt the presence of a parent affected a child's behaviour in the surgery. Attitudes towards a number of other practices such as 'tell-show-do', inhalation sedation, physical restraint, voice control and modelling were also sought. The investigators analysed the responses of 218 dentists and tried to determine if opinions and practices were affected by the respondent's age, gender or year of qualification.

One of the most notable findings was the respondents' positive attitude towards parental accompaniment. However, there were some reservations, with only $58 \%$ of dentists believing that children over the age of 3 years behaved better in the presence of a parent. This contrasted markedly with the $89 \%$ who felt that children under the age of 3 were more compliant when a parent was with them throughout their treatment. Interestingly, recently qualified dentists appeared the least keen on having a parent present during a child's treatment.

Another key finding from Crossley and Joshi's paper was the popularity of the well-established behavioural technique 'tellshow-do' with $87 \%$ of the sample citing this as their most favoured approach. Nitrous oxide inhalation sedation was also found to be a common adjunct to the behavioural armamentarium of the paediatric dentist. Respondents were far less comfortable, however, with the more authoritarian approaches with 73\% expressing unease about hand-over-mouth techniques and $69 \%$ stating that they were uncomfortable with the practice of active restraint. Interestingly, women were significantly less happy with active restraint than were their male colleagues. I wonder what $\mathrm{Dr}$ Freud would have said about that?

Dr Helen Rodd, Lecturer in Child Dental Health, School of Clinical Dentistry, University of Sheffield 